\title{
Hypoxia-induced ROS promotes mitochondrial fission and cisplatin chemosensitivity via $\mathrm{HIF}-1 \alpha / \mathrm{Mff}$ regulation
}

\author{
Kun $\mathrm{Wu}^{1}$, Yuan-yuan $\mathrm{Mao}^{3}$, Qi Chen ${ }^{4}$, Bolin Zhang ${ }^{5}$, Sheng Zhang, ${ }^{1, \#}$, Han-jiang Wu ${ }^{1, \# \text {, }}$ \\ Yan $\mathrm{Li}^{2, \#}$
}

1. Department of Stomatology, Second Xiangya Hospital, Central South University

2. Department of Oral and Maxillofacial-Head and Neck Oncology, Shanghai Ninth People's Hospital, Shanghai Jiao Tong University, School of Medicine

3. Department of Anesthesiology, Second Xiangya Hospital, Central South University

4. Department of Stomatology Qingpu Branch of Zhongshan Hospital, Fudan University, Shanghai, China

5. Department of Stomatology, Xinhua Hospital affiliated to Shanghai Jiao Tong University

\# Correspondence should be addressed to:

Yan Li, Department of Oral and Maxillofacial-Head and Neck Oncology, Shanghai Ninth People's Hospital, Shanghai Jiao Tong University, School of Medicine. Mailing address: No.639, Zhizaoju Road, Shanghai, 200011, China. Email address: liyan1106369@163.com.

Han-jiang Wu, Department of Stomatology, Second Xiangya hospital, Central South University, Renmin road, No. 139, Changsha, Hunan, 410011, China. Email address: wuhanjiang@csu.edu.cn

Sheng Zhang, Department of Stomatology, Second Xiangya hospital, Central South University, Renmin road, No. 139, Changsha, Hunan, 410011, China. Email address: drzhangsheng@csu.edu.cn

\begin{abstract}
Chemotherapy treatment based on Cisplatin (CDDP) is established as the drug of choice for head and neck squamous cell carcinoma (HNSCC). Malignant tumors respond to microenvironment alteration through a dynamic balance of mitochondrial fission and fusion. HNSCC is known to have hypoxic conditions, yet the effects and underlying mechanisms of hypoxia on chemosensitivity and mitochondrial dynamics remain unclear. We found that hypoxia promoted mitochondrial fission and CDDP sensitivity in HNSCC cells. Importantly, Mff was shown to be correlated with chemosensitivity in clinical samples of HNSCC that underwent a hypoxic condition. Hypoxia-inducible factor $1 \alpha$-subunit (HIF-1 $\alpha$ ) dramatically increased Mff transcriptional expression and directly bound to Mff. Hypoxia enhanced the release of reactive oxygen species (ROS) and upregulated the expression of Mff via HIF-1 $\alpha$ in HNSCC cells. ROS depletion in HNSCC cells attenuated HIF-1 $\alpha$, Mff expression, and mitochondrial fission. Moreover, a knockdown of Mff suppressed hypoxia-induced mitochondrial fission and decreased CDDP chemosensitivity in vivo and in vitro. Our findings revealed that the hypoxiainduced release of ROS promoted mitochondrial fission and CDDP chemosensitivity via the regulation of HIF-1 $\alpha / \mathrm{Mff}$ in HNSCC cells, indicating that Mff may serve as a new biomarker to predict neoadjuvant chemosensitivity in HNSCC patients.
\end{abstract}

Key words: hypoxia; cisplatin sensitivity; mitochondrial fission; ROS; head and neck squamous cell carcinoma 


\section{Introduction}

Head and neck squamous cell carcinoma (HNSCC), an aggressive and common malignant tumor causing high morbidity and mortality, is a major health challenge

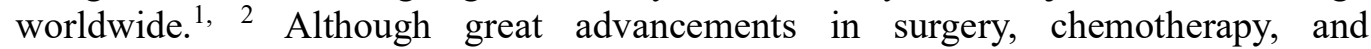
radiotherapy treatments have been achieved in the past few decades, the overall 5-years survival rate of HNSCC patients has remained approximately $50 \%{ }^{3}$ For HNSCC patients, cisplatin (CDDP) based chemotherapy has been established as the drug of choice ${ }^{4}$ However, chemoresistance continues to be responsible for adverse effects from HNSCC treatments, which has led to poor outcomes. Thus, a better understanding of the molecular mechanisms underlying chemoresistance is critical for establishing the most appropriate treatment strategies.

The role of the tumor microenvironment and hypoxia in cancer progression has garnered much attention in recent years. ${ }^{5,6}$ Normally, the $\mathrm{O}_{2}$ level of tissues ranges from $1 \%$ to $11 \%$, while cells are often cultured in a $20 \% \mathrm{O}_{2}$ environment. ${ }^{7}$ Rapid proliferation and unmatched angiogenesis in cancer cells leads to a hypoxic condition in most solid malignant tumors. ${ }^{8}$ A recent study has shown that HNSCC experiences hypoxic conditions, which may positively affect anti-EGFR therapies. ${ }^{9}$ Previous studies have also shown that excessive accumulation of reactive oxygen species (ROS) could promote cancer progression, ${ }^{10}$ and hypoxia induces increased ROS. ${ }^{8,}{ }^{11}$ To alter cellular adaptive responses to hypoxia, the activity of hypoxia-inducible factor $1 \alpha$-subunit (HIF-1 $\alpha$ ) acts as a key mechanism. ${ }^{12}$ HIF-1 $\alpha$, a transcription factor, could transactivate various target genes to participate in malignant tumor biological behavior. ${ }^{13,14}$

Previous studies have shown that abnormal mitochondrial dynamics are correlated with diseases via the regulation of apoptosis. ${ }^{15,16}$ Tumor cells respond to microenvironment alteration through the dynamic balance of mitochondrial fission and fusion. ${ }^{17}$ Accumulated evidence has revealed that mitochondrial fission promotes tumor cell CDDP sensitivity in tongue squamous cell carcinoma. ${ }^{18}{ }^{19}$ However, how mitochondrial fission contributes to CDDP sensitivity in hypoxia-cultured HNSCC cells is still unclear. Furthermore, the molecular mechanism of mitochondrial fission and fusion in modulating CDDP sensitivity in hypoxic-cultured HNSCC cells has not been elucidated.

In the present study, we aimed to investigate the effect of hypoxia on CDDP-cultured HNSCC cells and mitochondrial fission. This study revealed that hypoxia-induced ROS accumulation promotes mitochondrial fission and CDDP chemosensitivity via the regulation of HIF-1 $\alpha /$ Mff in HNSCC cells.

\section{Materials and Methods \\ Ethics}

The study was approved by the Ethics Committee of the Second Xiangya Hospital (Approval No. 2020530, 2020/9/14), and written informed consent was obtained from all participants prior to enrollment. All experimental methods abided by the Helsinki Declaration. All animal studies were performed in accordance with the NIH Guide for Care and Use of Laboratory Animals and were approved by the Second Xiangya Hospital Animal Ethical and Welfare Committee (Approval No. 2020489, 2020/9/9).

\section{Patients and Specimens}

All HNSCC samples were obtained from the Department of Oral and Maxillofacial Surgery at the Second Xiangya Hospital of Central South University. More specifically, 
72 pairs of tumor and adjacent normal tissues were collected from primary HNSCC patients who underwent an initial surgical treatment between July 2017 and June 2019. Patients who received preoperative treatment and had a malignant history were excluded. Clinical parameters were obtained from medical records. Moreover, clinical stage and pathological differentiation were performed according to the World Health Organization Classification of Tumors and the tumor node metastasis staging system from the Union for International Cancer Control.

\section{Cell cultures and reagents}

HN6 and HN30 were kindly provided by the University of Maryland Dental School, USA. Cal27 and 293T cells were purchased from the American Type Culture Collection (MD, USA). Cal27, HN6, HN30, and 293T cells were cultured in Dulbecco's modified Eagle's medium (Gibco-BRL, CA, USA) supplemented with $10 \%$ fetal bovine serum (Gibco-BRL, CA, USA), streptomycin $(100 \mu \mathrm{g} / \mathrm{mL})$, and penicillin $(100 \mathrm{units} / \mathrm{mL})$ at $37^{\circ} \mathrm{C}$ in a humidified $5 \% \mathrm{CO}_{2}$ atmosphere. For hypoxia, tumor cells were cultured in a tri-gas incubator (ESCO, Singapore), consisting of $5 \% \mathrm{CO}_{2}, 94 \% \mathrm{~N}_{2}$, and $1 \% \mathrm{O}_{2}$. Hydrogen peroxide $\left(\mathrm{H}_{2} \mathrm{O}_{2}\right), \mathrm{CDDP}$, and $\mathrm{N}$-acetyl-L-cysteine (NAC) were obtained from Sigma-Aldrich (USA).

\section{RNA extraction and real-time PCR analysis}

The RNA was extracted from the tumor samples and cells using TRIzol reagent (Takara, Japan), and cDNA was synthesized with a PrimeScript RT Reagent Kit (Takara, Japan). A real-time PCR (RT-PCR) was performed using the SYBR Premix Ex Taq Reagent Kit (Takara, Japan) with the StepOne RT-PCR System (Life Technologies, USA) according to the manufacturer's instructions. The mRNA levels were normalized to $\beta$ actin levels. The primer sequences used in the present study are listed in Supplementary Table 1.

\section{MTT assays}

Cell viability was assessed by the 3-(4,5-dimethylthiazol-2-yl)-2,5-diphenyltetrazolium bromide (MTT) assay. Tumor cells were seeded in a 96-well plate at a density of 2000 cells per well with $100 \mu \mathrm{L}$ of medium at $37^{\circ} \mathrm{C}$. The cells were incubated with $100 \mu \mathrm{L}$ of $0.5 \mathrm{mg} / \mathrm{mL}$ MTT (Sigma-Aldrich, USA) in Dulbecco's modified Eagle's medium for $4 \mathrm{~h}$. Twelve hours later, the cells were cultured with a gradient concentration of CDDP for $48 \mathrm{~h}$. The formazan was solubilized in $150 \mu \mathrm{L}$ dimethyl sulfoxide. Absorbance was measured using a multi-well plate reader (Bio-Rad Laboratories, Hercules, CA, USA) at $490 \mathrm{~nm}$. The half maximal inhibitory concentration (IC50) was used to evaluate the degree of tumor cell response to CDDP.

\section{Apoptosis analysis}

Apoptosis was assessed by flow cytometric analysis and terminal deoxynucleotidyl trans-ferase-mediated dUTP nick end labeling (TUNEL). TUNEL assays were performed using the Click-i ${ }^{\mathrm{TM}}$ Plus TUNEL assay kit (Invitrogen, USA) according to the manufacturer's instructions. Flow cytometric analysis was performed using the Annexin V FITC Apop Dtec Kit (BD Pharmingen, USA) according to the manufacturer's instructions. The cells were trypsinized and centrifuged at $300 \times \mathrm{g}$ for 10 min at $4^{\circ} \mathrm{C}$. Afterward, the cell pellets were resuspended in binding buffer and stained with Annexin V FITC and propidium iodide for $15 \mathrm{~min}$ on ice. Apoptotic cells were analyzed by flow cytometry (BD FACS Calibur, USA). 


\section{Western blot analysis}

Tumor cells were harvested at the indicated times in SDS lysis buffer (Beyotime, China). Protein samples were separated using $10 \%$ polyacrylamide gels and transferred to 0.45 $\mu \mathrm{m}$ poly (vinylidene fluoride) membranes (Merck Millipore, USA). The membranes were soaked in $10 \%$ skim milk for $1 \mathrm{~h}$ at room temperature and incubated overnight at $4^{\circ} \mathrm{C}$ with the primary antibody. Subsequently, the membranes were probed with horseradish peroxidase-labeled secondary rabbit antibodies (Beyotime, China) for $1 \mathrm{~h}$. The electrochemiluminescence (New Cell Molecular Biotech, China) developing solution was used to detect signals on a chemiluminometer (Amersham Imager 600). $\beta$-Actin was used as a control. The antibodies (1:1000) against Mff, HIF-1 $\alpha$, Mfn2 and $\beta$-actin were purchased from Cell Signaling Technology (USA).

\section{Detection of relative ROS levels}

Intracellular ROS levels were detected using 2,7-dichloro-dihydrofluorescein diacetate (Sigma-Aldrich, USA) and dihydroethidium (Sigma-Aldrich, USA) by flow cytometry (BD FACS Calibur, USA). This ROS analysis technique has been described in a previous study. ${ }^{8}$

\section{Mitochondrial staining and analysis of mitochondrial fission}

The cells were cultured on coverslips and stained with MitoSpy ${ }^{\mathrm{TM}}$ Red CMXRos (BioLegend, USA) for $30 \mathrm{~min}$ at $37^{\circ} \mathrm{C}$. Afterward, the cells were fixed with $4 \%$ paraformaldehyde and stained with 4'-6-diamidino-2-phenylindole (DAPI; Sangon Biotech, China). Cells were viewed using a TCS SP2 laser-scanning confocal microscope (Leica Microsystems, Germany). Mitochondrial morphology was then quantified and assessed as described previously. ${ }^{8}$

\section{Analysis of immunofluorescence}

The cells grown on coverslips were fixed with $4 \%$ paraformaldehyde, permeabilized with $0.1 \%$ Triton $\mathrm{X}-100$, blocked with $10 \%$ goat serum, and incubated with the Mff antibody (CST, 1:500) and the HIF- $1 \alpha$ antibody (CST, 1:500) at $4^{\circ} \mathrm{C}$ overnight then incubated with the Alexa Fluor 488-cojugated secondary antibody (Invitrogen, USA) or Alexa Fluor 549-cojugated secondary antibody (Invitrogen, USA) at room temperature in the dark. Cells were then stained with DAPI (Sangon Biotech, China) to detect the nuclei and viewed using a TCS SP2 laser-scanning confocal microscope (Leica Microsystems, Germany).

\section{Co-immunoprecipitation analysis}

A co-immunoprecipitation (Co-IP) analysis was performed in our previous study. ${ }^{20} \mathrm{In}$ brief, the cells were lysed with IP buffer and incubated with the specific antibodies previously mentioned at $4^{\circ} \mathrm{C}$ overnight, followed by incubation with protein $\mathrm{A} / \mathrm{G}$ Magbeads (Merck) for $6 \mathrm{~h}$ at $4^{\circ} \mathrm{C}$. The proteins were separated from the beads for 10 min at $105^{\circ} \mathrm{C}$. The supernatants were then used for subsequent immunoblotting analyses.

\section{Cell transfection}

SiRNA (Ribobio, China) was transfected into 293T cells using Lipofectamine RNAiMAX (Invitrogen, USA) according to the manufacturer's instructions. Twentyfour hours later, the cells were harvested and the corresponding experiments were performed. The siRNAs used in the present study were designed and synthesized by Guangzhou RiboBio Co., Ltd. (Guangzhou, China). 


\section{Luciferase analysis}

In brief, the $293 \mathrm{~T}$ cells $\left(2 \times 10^{5}\right)$, which were cultured on a 6 -well plate, were cotransfected with Mff response luciferase plasmid and Renilla luciferase vector using the Lipofectamine 3000 reagent (Invitrogen, USA), according to the manufacturer's instructions. After $48 \mathrm{~h}$, the dual luciferase system (Promega, USA) was used to detect luciferase activity.

\section{Lentiviral transduction and stable strain screening}

The Mff interfering lentiviral vector ${ }^{21}$ (LV-Mff) and interfering control lentiviral vector (LV-NC), which were constructed by HanYin Biotechnology Co, Ltd (China), conferred puromycin resistance. Lentiviral transduction was performed according to the manufacturer's instructions. After $72 \mathrm{~h}$ of transfection, the culture medium with puromycin at a final concentration of $10 \mu \mathrm{g} / \mathrm{mL}$ was used to screen for stably stained cells.

\section{Xenograft mouse experiments}

The animal experiments, using BALB/C nude mice (Shanghai Laboratory Animal Center) aged 4 weeks old, were performed in accordance with the ethical standards and national guidelines. Cal27 cells $\left(1 \times 10^{6}\right)$ stably expressing LV-Mff or LV-NC were resuspended in $100 \mu \mathrm{l}$ of phosphate buffered saline and subcutaneously injected into the dorsal flanks of mice ( $\mathrm{n}=6$ in each group). Tumor sizes were measured using a caliper every three days. CDDP and saline were intraperitoneally injected at $5 \mathrm{mg} / \mathrm{kg}$ body weight in every 3 days from day 7 to 33. After day 33, the mice were sacrificed to collect tumor samples and measure tumor weights.

\section{Statistical analysis}

To compare the differences in expression between HNSCC and matched adjacent normal tissue, paired t-tests were performed. The Kruskal-Wallis tests and MannWhitney $U$ tests were used to analyze the association between mRNA (SLC2A1, PDGFB, VEGFA and HIF- $1 \alpha$ ) levels and clinical parameters. The comparison between the groups was analyzed using one-way ANOVA or Student's t-test. The correlation between two variables was analyzed using Pearson's correlation. All statistical analyses were performed using SPSS 19.0 (SPSS, Chicago, IL, USA). All values were two-sided, and $\mathrm{P}<0.05$ was considered statistically significant.

\section{Results}

\section{Hypoxia facilitates CDDP chemosensitivity in HNSCC cells}

Hypoxia can regulate malignant tumor vascularization, invasion, chemoresistance, and metastasis in various types of cancer. ${ }^{8,22,23}$ To understand whether the HNSCC microenvironment was hypoxic, hypoxia markers ${ }^{8}$ (solute carrier family 2 member 1 (SLC2A1), platelet-derived growth factor B (PDGFB), vascular endothelial growth factor A (VEGFA), and HIF-1 $\alpha$ ) were detected in 72 samples of tumor tissues paired with adjacent normal tissues. The expression levels of hypoxia markers were significantly higher in tumor tissues than in the normal tissues (Fig. 1A). The levels of PDGFB were related to patient age ( $\geq 60$ years and $<60$ years), sex (male and female), smoking history (yes or no), and alcohol history (yes or no) in our study (Supplementary Table 2). An analysis of The Cancer Genome Atlas (TCGA) database further showed that these hypoxia markers were also significantly upregulated in HNSCC samples (Supplementary Fig. 1A). These data confirm the hypoxic environment in HNSCC tumors. 

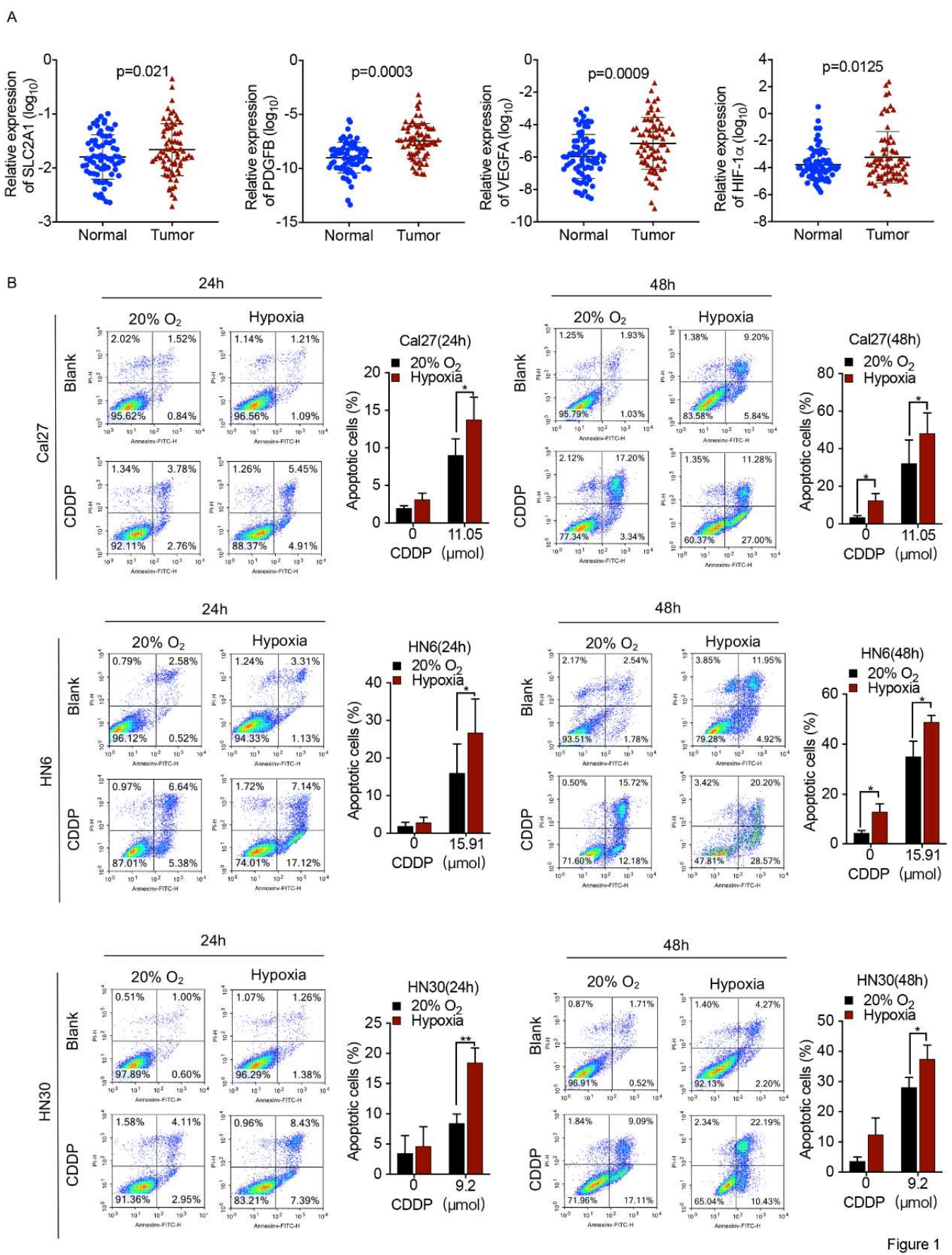

Figure 1. Hypoxia induces CDDP chemosensitivity in HNSCC cells

(A) The expression of hypoxia markers (SLC2A1, PDGFB, VEGFA, and HIF-1 $\alpha$ ) was upregulated in HNSCC samples ( $\mathrm{n}=72)$.

(B) Hypoxia promotes cell apoptosis in Cal27, HN6, and HN30 cells, while the apoptotic effect of hypoxia was augmented in HNSCC cells after incubation with CDDP. Cal27, HN6, and HN30 cells were treated with the indicated concentrations of CDDP. Data are presented as the mean \pm standard deviation (SD) from 3 independent experiments $(* \mathrm{P}<0.05, * * \mathrm{P}<0.01)$.

To determine the effect of hypoxia on CDDP sensitivity in HNSCC, Cal27, HN6, and $\mathrm{HN} 30$ cells were cultured in $20 \% \mathrm{O}_{2}$ or a hypoxic environment $\left(1 \% \mathrm{O}_{2}\right)$. The IC50 value of CDDP, identified by MTT assay in Cal27, HN6, and HN30 cells, was 11.05, 
15.91, and 9.2 $\mu \mathrm{M}$, respectively (Supplementary Fig. 1B). Cal27, HN6, and HN30 cells were treated with the IC50 of CDDP for apoptosis assays. The proportion of apoptotic cells (positive for Annexin V FITC and propidium iodide staining) in hypoxia-cultured HNSCC cells was higher than that in $20 \% \mathrm{O}_{2}$-cultured HNSCC cells. Moreover, the apoptotic effect of hypoxia was augmented in HNSCC cells after incubation with CDDP. The proportion of apoptotic cells in hypoxia-CDDP-cultured HNSCC cells was dramatically higher than that in the $20 \% \mathrm{O}_{2}$-CDDP-cultured HNSCC cells after $24 \mathrm{~h}$ and $48 \mathrm{~h}$ (Fig. 1B). These findings showed that hypoxia enhances CDDP sensitivity in HNSCC cells.

\section{Hypoxia increases mitochondrial fission via Mff/Mfn2 and ROS levels in HNSCC cells}

In the tumor microenvironment, ROS increases under hypoxic conditions ${ }^{8,11}$ and is produced as a byproduct by mitochondria. ${ }^{10}$ To explore the effect of hypoxia on ROS and mitochondrial fission, ROS levels and mitochondrial fission were measured in Cal27, HN6, and HN30 cells. After $24 \mathrm{~h}$ or $48 \mathrm{~h}$ cultured in hypoxic conditions, Cal27, $\mathrm{HN} 6$, and $\mathrm{HN} 30$ cells showed a dramatic increase in both the intracellular $\mathrm{H}_{2} \mathrm{O}_{2}$ and superoxide $\left(\mathrm{O}_{2}^{-}\right)$levels compared to the $20 \% \mathrm{O}_{2}$-cultured cells (Fig. 2A). Mitochondrial fission in hypoxia-cultured HNSCC cells increased more dramatically than that in $20 \% \mathrm{O}_{2}$-cultured HNSCC cells for $24 \mathrm{~h}$ or $48 \mathrm{~h}$, indicated by decreased mitochondrial length (Fig. 2B).

To clarify the molecular mechanism by which hypoxia-induced mitochondrial fission occurs, the major mitochondrial fission and fusion genes (Drp1, Fis1, Mff, Mid49, Mid51, GDAP1, Mfn1, Mfn2 and OPA1) ${ }^{24}$ expression levels were detected using RTPCR in Cal27, HN6, and HN30 cells (Supplementary Fig 1C). Our results showed that the transcriptional expression level of Mff increased more significantly in hypoxiacultured HNSCC cells than that in $20 \% \mathrm{O}_{2}$-cultured HNSCC cells, and also showed a time-dependent increasing pattern (Fig. 2C). In addition, the expression level of Mfn2 decreased more dramatically in hypoxia-cultured HNSCC cells compared to that in $20 \%$ $\mathrm{O}_{2}$-cultured HNSCC cells (Fig. 2C). To further determine if Mff and Mfn2 proteins were responsible for mitochondrial fission induced by hypoxia, Mff and Mfn2 were detected by western blotting. The post-translational level of Mff increased under hypoxia in a time-dependent manner (Fig. 2D). The post-translational level of Mfn2, however, decreased under hypoxic conditions (Fig. 2D). To confirm hypoxic conditions in HNSCC cells, the protein level of HIF-1 $\alpha$, detected by western blotting, was significantly increased in HNSCC cells under hypoxic conditions (Fig. 2D). These results indicate that hypoxic HNSCC cells undergo mitochondrial fission via Mff upregulation and Mfn2 downregulation.

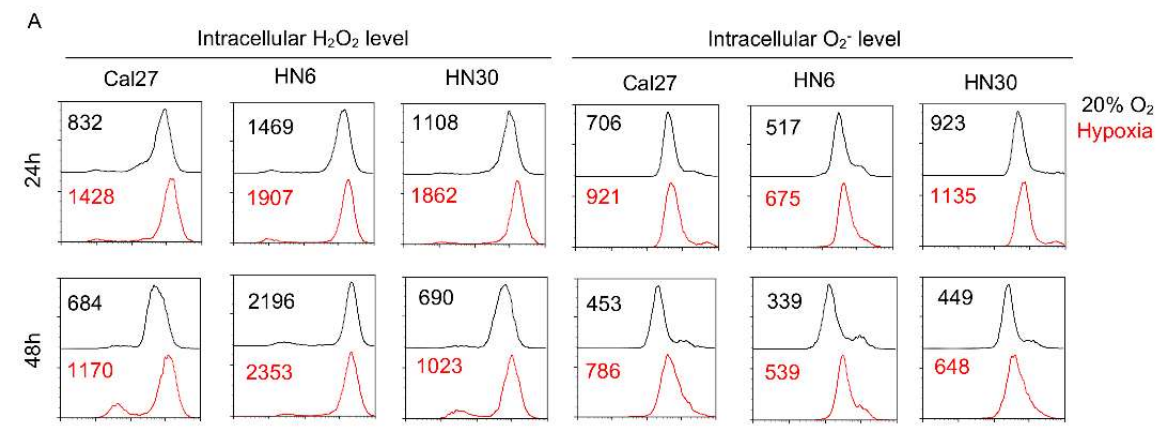




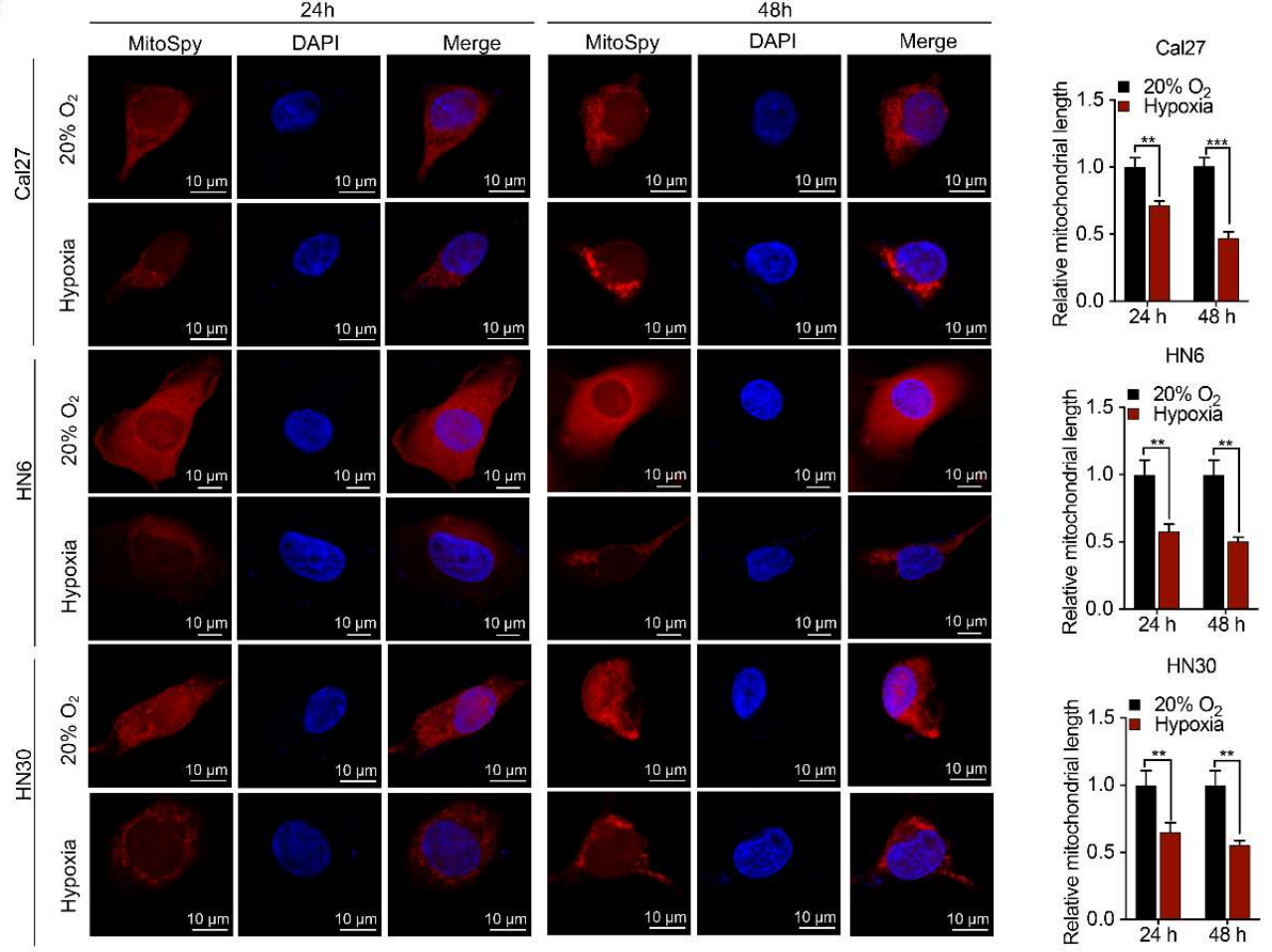

C

Hypoxia

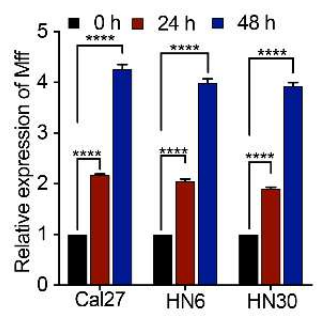

Hypoxia

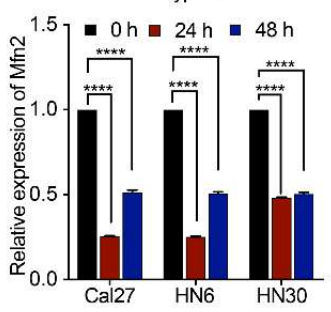

D

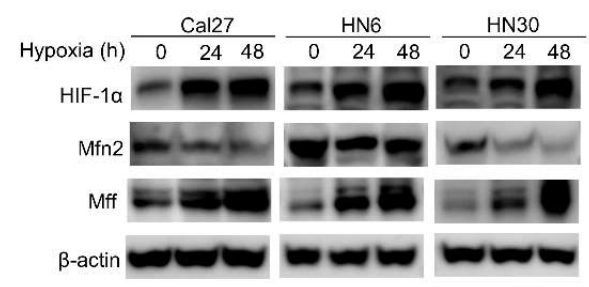

Figure 2. Hypoxia increases ROS levels and mitochondrial fission via Mff and Mfn2 in HNSCC cells

(A) The intracellular ROS levels $\left(\mathrm{O}_{2}-\right.$ and $\left.\mathrm{H}_{2} \mathrm{O}_{2}\right)$ of Cal27, HN6, and $\mathrm{HN} 30$ cells after $24 \mathrm{~h}$ or $48 \mathrm{~h}$ of exposure to hypoxia were measured.

(B) The mitochondrial lengths of Cal27, HN6, and HN30 cells were measured using MitoSpy staining.

(C) The Mff and Mfn2 were screened by RT-PCR for hypoxia-induced mitochondrial fission in HNSCC cells.

(D) Western blot analysis was used to confirm the protein expression of HIF-1 $\alpha$, Mff, and Mfn2 in Cal27, HN6, and HN30 cells. Data are presented as the mean \pm SD from 3 independent experiments $(* * \mathrm{P}<$ $0.01, * * * \mathrm{P}<0.001, * * * * \mathrm{P}<0.0001)$.

\section{Hypoxia enhances ROS release and promotes mitochondrial fission via Mff in} HNSCC cells treated with CDDP

To explore the effect of hypoxia on ROS and mitochondrial fission in HNSCC cells treated with CDDP, ROS levels and mitochondrial fission were measured. In hypoxiaCDDP-cultured HNSCC cells, the intracellular $\mathrm{H}_{2} \mathrm{O}_{2}$ levels and superoxide $\left(\mathrm{O}_{2}^{-}\right)$levels were significantly higher than that in $20 \% \mathrm{O}_{2}$-CDDP-cultured cells for $24 \mathrm{~h}$ or $48 \mathrm{~h}$ (Fig. 3A). The mitochondrial fission in hypoxia-CDDP-cultured HNSCC cells were more dramatically increased than that in $20 \% \mathrm{O}_{2}-\mathrm{CDDP}$-cultured HNSCC cells for 24 h or $48 \mathrm{~h}$ (Fig. 3B).

The protein level of HIF-1 $\alpha$ significantly increased in the hypoxia-CDDP-cultured 
HNSCC cells (Fig. 3C). To determine if Mff and Mfn2 proteins were still responsible for mitochondrial fission induced by hypoxia in CDDP-treated cells, Mff and Mfn2 were detected by western blotting. The post-translational level of Mff increased in hypoxia-CDDP-cultured HNSCC cells and showed a time-dependent pattern, while the post-translational level of Mfn2 was not significantly altered (Fig. 3C). Together, these results suggest that hypoxia-CDDP-cultured HNSCC cells undergo mitochondrial fission through Mff upregulation.
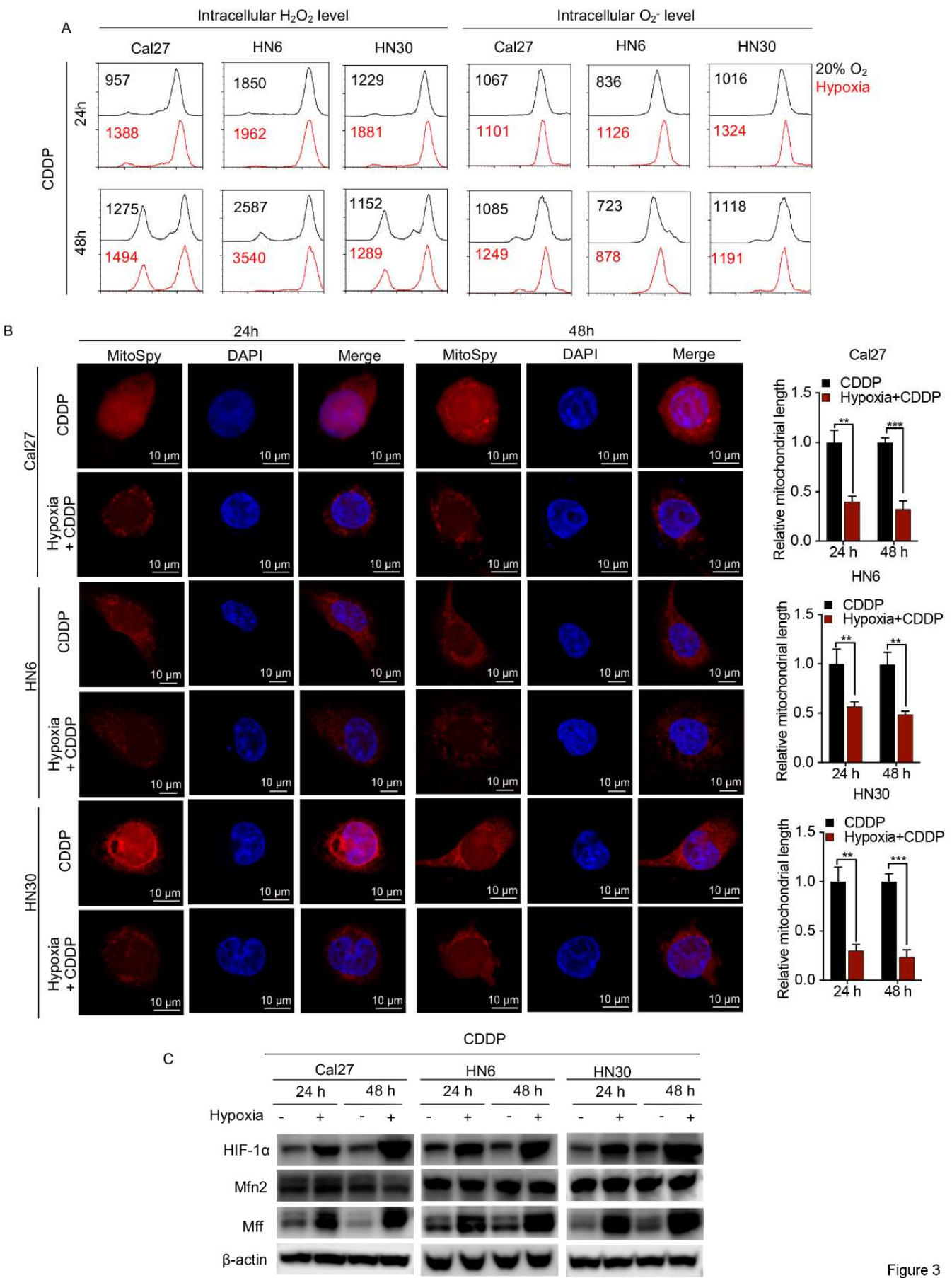

Figure 3. Hypoxia enhances ROS levels and mitochondrial fission via Mff in HNSCC cells treated with CDDP 
(A) The intracellular ROS levels $\left(\mathrm{O}_{2}-\right.$ and $\left.\mathrm{H}_{2} \mathrm{O}_{2}\right)$ of hypoxia-CDDP-cultured HNSCC cells were measured by flow cytometric analysis.

(B) Cal27, HN6, and HN30 cells stained with MitoSpy to evaluate mitochondrial length by confocal microscopy.

(C) Western blot analysis was used to confirm the protein expression of HIF-1 $\alpha$, Mff, and Mfn2 in Cal27, HN6, and HN30 cells. Data are presented as the mean \pm SD from 3 independent experiments $(* * \mathrm{P}<$ $0.01, * * * \mathrm{P}<0.001)$.

\section{Hypoxia-induced HIF-1 $\alpha$ facilitates Mff expression level in HNSCC}

To examine whether Mff accounts for hypoxia-induced chemosensitivity and malignant transformation in HNSCC, the expression levels of HIF-1 $\alpha$, Mff, and Mfn2 were detected in HNSCC tissues. The mRNA expression level of Mff was upregulated more in CDDP-sensitive HNSCC patients than in CDDP-resistant HNSCC patients, while Mfn2 expression was not significantly altered (Fig. 4A). Further analysis revealed that HIF-1 $\alpha$ expression was correlated with the Mff levels $(\mathrm{r}=0.9456, \mathrm{P}<0.0001)$ but not correlated with Mfn2 (Fig. 4B). Moreover, both Mff and Mfn2 expression were significantly upregulated in HNSCC tissues (Fig. 4C). In addition, Mff was correlated with the extracapsular spread of metastatic lymph nodes, while Mfn2 was related to tumor size, prior radiotherapy, and pathological differentiation (Fig. 4D). Together, these data revealed that hypoxia-induced mitochondrial fission events in HNSCC are correlated with chemosensitivity.

In the present study, a significantly positive correlation between HIF-1 $\alpha$ and Mff was observed. This result indicated that HIF-1 $\alpha$ may interact and bind directly with Mff in HNSCC cells. To verify this hypothesis, co-IP, luciferase analysis, and immunofluorescence staining were performed. The endogenous interaction between HIF-1 $\alpha$ and Mff was confirmed by Co-IP (Fig. 4E). Moreover, hypoxia-induced HIF$1 \alpha$ dramatically increased the Mff transcriptional activity in 293T cells, and the knockdown of HIF-1 $\alpha$ partly decreased Mff activity (Fig. 4F). In addition, immunofluorescent staining showed that Mff was localized to the cytoplasm and that part of HIF-1 $\alpha$ was also localized to the cytoplasm (Fig. 4G). These results suggest that HIF-1 $\alpha$ binds directly and regulates Mff in the cytoplasm of HNSCC cells.

A
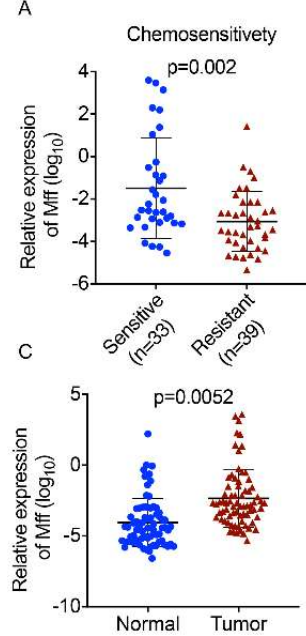
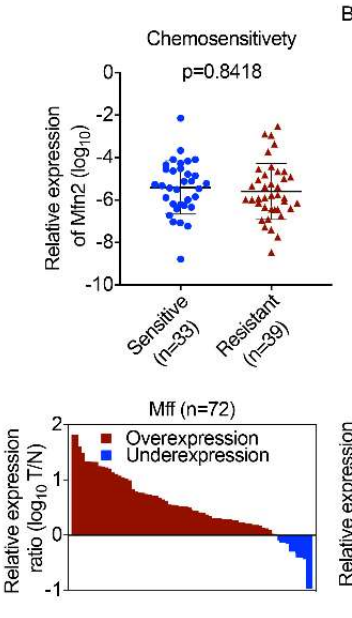
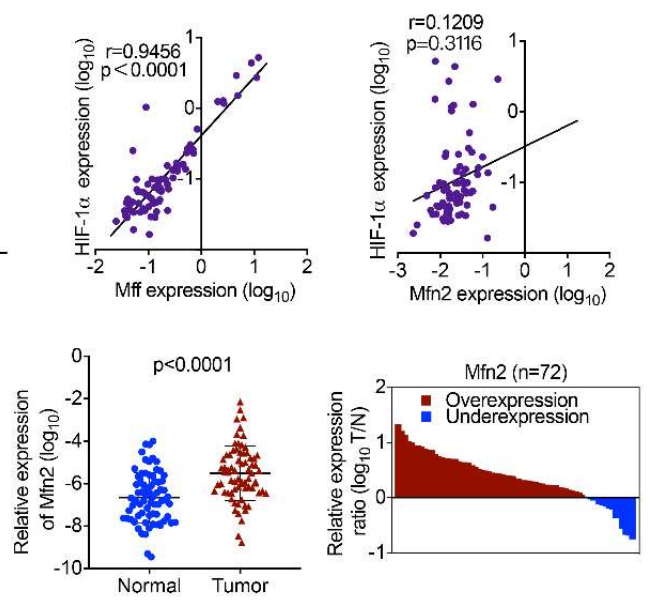

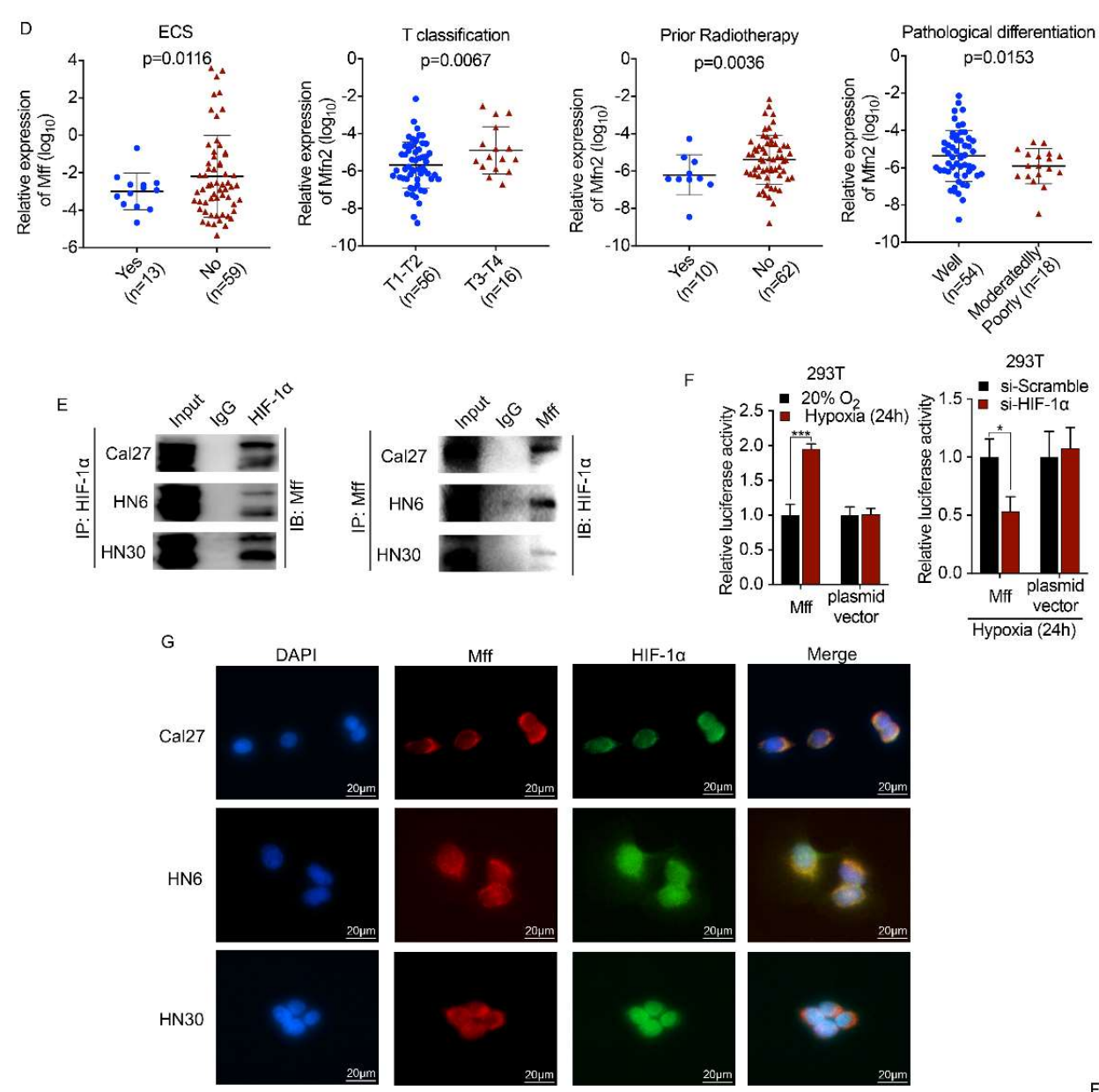

Figure 4

Figure 4. HIF-1 $\alpha$ promotes Mff expression level in HNSCC

(A) The level of Mff expression was significantly upregulated in HNSCC patients with CDDP sensitivity.

(B) The remarkably positive association between Mff expression and HIF-1 $\alpha$ expression was found by expression correlation analysis.

(C) Both Mff and Mfn2 expression were upregulated more in HNSCC tissues than in normal tissues.

(D) The correlation between clinical parameters and Mff and Mfn2 was determined by correlation analysis.

(E) The interaction between Mff and HIF-1 $\alpha$ was determined via a co-immunoprecipitation assay.

(F) Hypoxia-induced HIF-1 $\alpha$ considerably increased the luciferase activity of Mff, and pretreatment with si-HIF-1 $\alpha$ partly inhibited this effect.

(G) The subcellular localization of Mff and HIF-1 $\alpha$ in the Ca127, HN6, and HN30 cells was assessed by immunofluorescence staining. Data are presented as the mean \pm SD from 3 independent experiments (* $\mathrm{P}<0.05, * * * \mathrm{P}<0.001)$

\section{ROS promote Mff upregulation via HIF-1 $\alpha$ in hypoxic-CDDP-cultured HNSCC cells}

In the present study, the findings showed that hypoxia increases ROS and HIF-1 $\alpha$ levels in HNSCC cells. To determine the effect of hypoxia-induced ROS on mitochondrial fission, $\mathrm{H}_{2} \mathrm{O}_{2}$ was treated exogenously to $20 \% \mathrm{O}_{2}$-cultured $\mathrm{HNSCC}$ cells and the free radical scavenger NAC was applied to hypoxic-CDDP-cultured HNSCC cells.

Hypoxia-induced ROS levels and mitochondrial fission were attenuated by NAC (1 $\mathrm{mM}, 6 \mathrm{~h})^{8}$ (Fig. 5A, B). The increase in HIF-1 $\alpha$ and Mff expression by hypoxia was restored by NAC treatment (Fig. 5C). Next, exogenous $\mathrm{H}_{2} \mathrm{O}_{2}(20 \mu \mathrm{M}, 6 \mathrm{~h})^{8}$ promoted 
mitochondrial fission (Supplementary Fig. 2A). In addition, $\mathrm{H}_{2} \mathrm{O}_{2}$ treatment increased HIF- $1 \alpha$ and Mff content in 20\% $\mathrm{O}_{2}$-cultured HNSCC cells (Supplementary Fig. 2B). These results showed that hypoxia-elicited ROS regulate the Mff protein level via HIF$1 \alpha$ to promote mitochondrial fission.
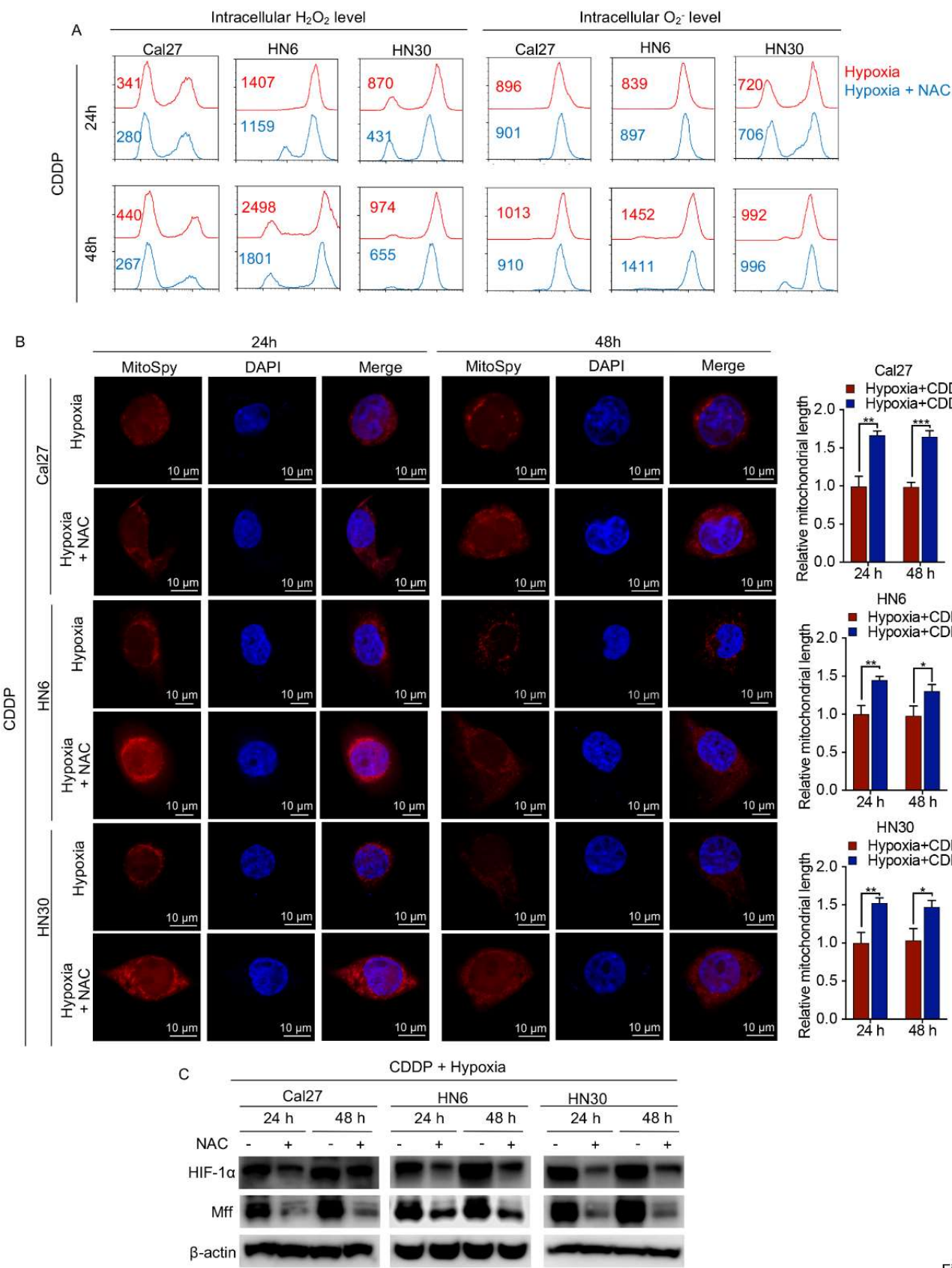

Figure 5. NAC attenuated hypoxia-upregulated HIF-1 $\alpha$ and Mff

(A-B) NAC decreased hypoxia-induced ROS and mitochondrial fission in HNSCC cells treated with CDDP.

(C) Western blot analysis was used to determine the protein expression of HIF-1 $\alpha$ and Mff in hypoxiaCDDP-cultured HNSCC cells treated with NAC. Data are presented as the mean \pm SD from 3 independent experiments $\left(* \mathrm{P}<0.05,{ }^{* *} \mathrm{P}<0.01,{ }^{* * *} \mathrm{P}<0.001\right)$. 


\section{Knockdown of Mff suppresses hypoxia-induced mitochondrial fission and CDDP chemosensitivity in HNSCC cells}

To evaluate the biological function of Mff knockdown in hypoxia-CDDP-cultured HNSCC cells, Cal27, HN6, and HN30 cells were stably transfected with LV-Mff and LV-NC. The protein expression level of Mff in HNSCC cells with LV-Mff was notably low (Supplementary Fig. 3A). Moreover, there was no statistically significant difference in the proportion of LV-Mff and LV-NC transfected HNSCC cells that were apoptotic in $20 \% \mathrm{O}_{2}$ for $24 \mathrm{~h}$ (Supplementary Fig. 3B). Sensitivity to CDDP was higher in the LV-NC transfected HNSCC cells than that in the LV-Mff transfected HNSCC cells under hypoxia for $24 \mathrm{~h}$ (Fig. 6A) and $48 \mathrm{~h}$ (Fig. 6B). Meanwhile, mitochondrial fission in hypoxia-CDDP-cultured conditions was dramatically decreased in HNSCC cells with LV-Mff for 24 or $48 \mathrm{~h}$ (Fig. 6C). Therefore, inhibition of hypoxia-induced mitochondrial fission could attenuate the sensitivity of hypoxic HNSCC cells to CDDP.

A
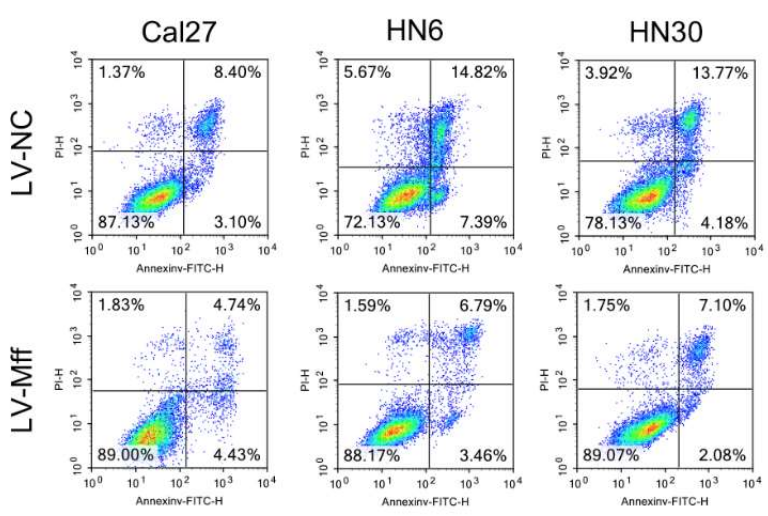

CDDP + Hypoxia (24h)

B
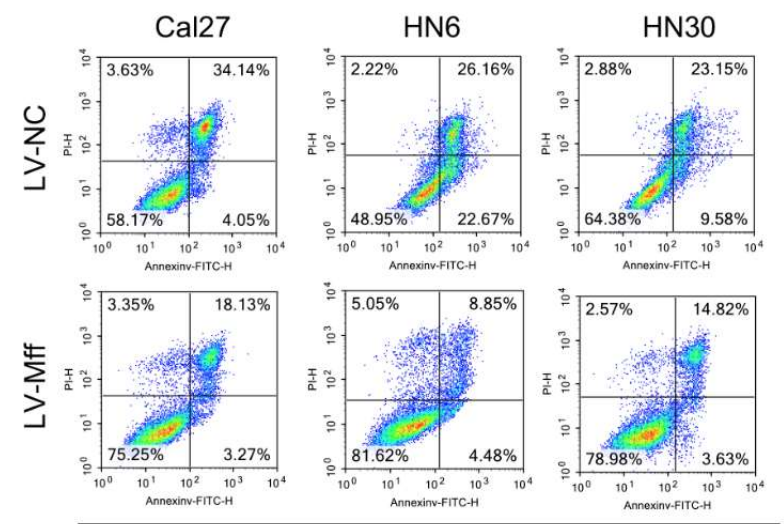

CDDP + Hypoxia (48h)
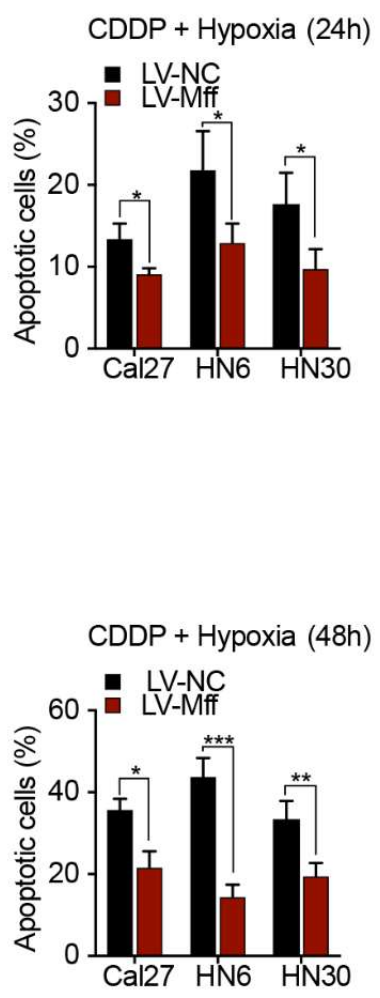


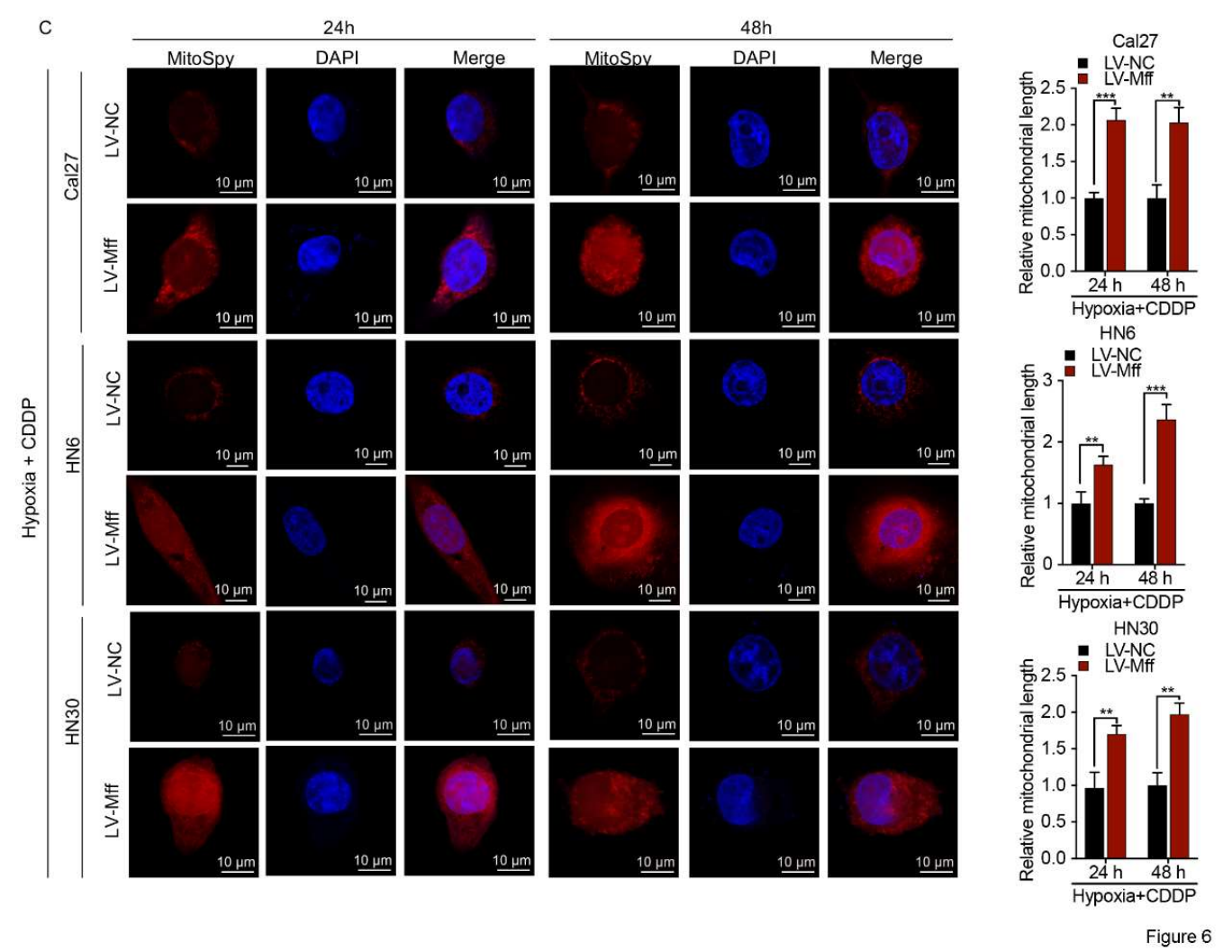

Figure 6. Knockdown of Mff attenuates CDDP chemosensitivity and mitochondrial fission in hypoxic conditions

(A-B) Flow cytometric analysis was used to analyze the proportion of HNSCC cells stably transfected with LV-Mff or LV-NC under hypoxic conditions.

(C) The mitochondrial length of HNSCC cells stably transfected with LV-Mff or LV-NC under hypoxic CDDP-cultured conditions were measured using MitoSpy staining. Data are presented as the mean $\pm \mathrm{SD}$ from 3 independent experiments $(* \mathrm{P}<0.05, * * \mathrm{P}<0.01, * * * \mathrm{P}<0.001)$.

\section{Mff regulates CDDP sensitivity in HNSCC xenografts}

Since Mff expression facilitates the chemosensitivity of HNSCC cells under hypoxia, we evaluated the effect of Mff on tumorigenicity in a xenograft model. For this purpose, Ca127 cells stably transfected with LV-Mff or LV-NC were injected subcutaneously into the dorsal flanks of nude mice. The results showed that Cal27 cells with a stable expression of LV-Mff had enhanced tumor growth and tumor weight in the presence of CDDP (Fig. 7A, B). Moreover, apoptosis was attenuated in CDDP-treated xenografts of Cal27 cells stably transfected with LV-Mff (Fig. 7C). Conclusively, our findings reveal that hypoxia-induced ROS promote mitochondrial fission and CDDP chemosensitivity through HIF-1 $\alpha /$ Mff regulation in HNSCC cells (Figure 7D). 

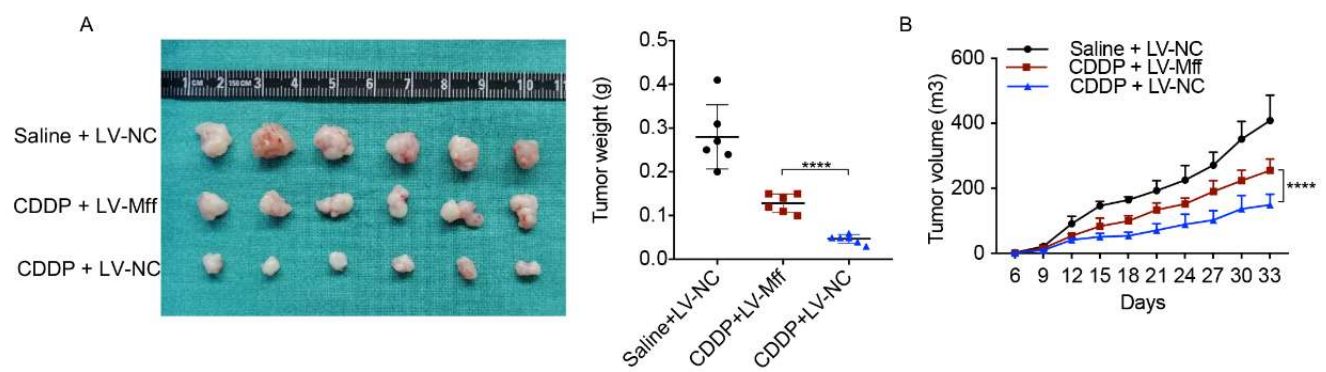

C
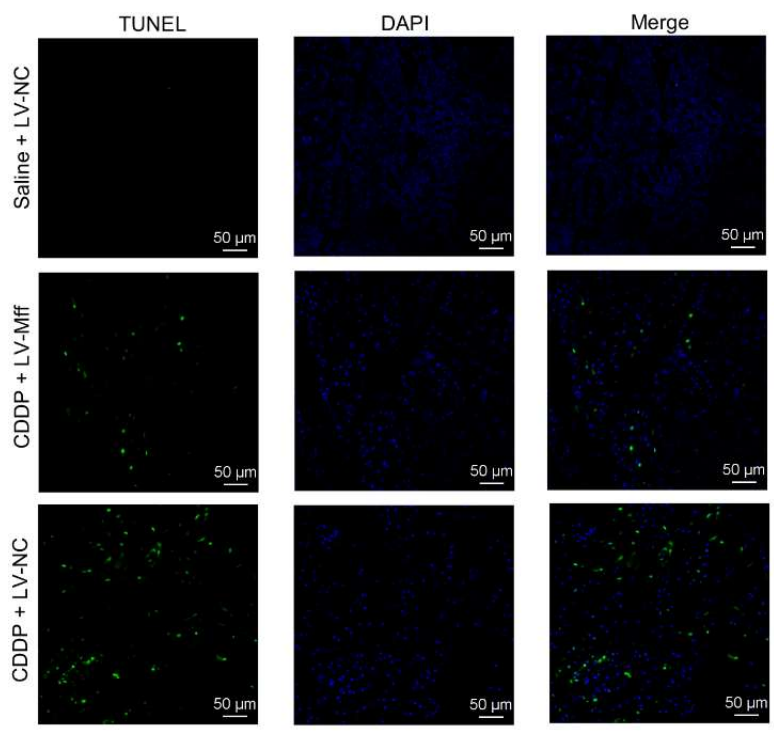

D

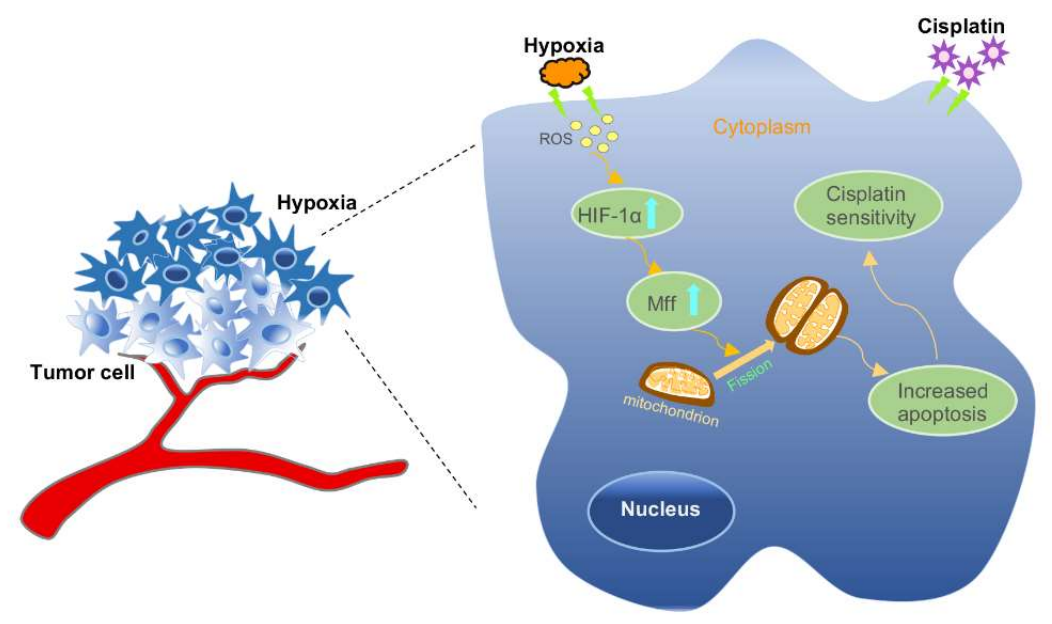

Figure 7

Figure 7. Mff knockdown inhibits apoptosis and CDDP sensitivity in HNSCC cells in vivo

(A) The tumor weights and volumes of Cal27 cells are shown. Nude mice bearing xenografts of Cal27 cells stably transfected with LV-NC or LV-Mff treated with saline or CDDP ( $n=6$ per group).

(B) Tumor growth curves of Cal27 cells for each group are shown.

(C) Tumor cell apoptosis in response to CDDP was attenuated in Cal27 cells stably transfected with LVMff.

(D) A proposed model illustrating the regulatory role of hypoxia-induced ROS promoting mitochondrial fission and CDDP chemosensitivity via regulated HIF-1 $\alpha / \mathrm{Mff}$. 


\section{Discussion}

The present study shows that Mff promotes mitochondrial fission and consequently CDDP sensitivity. Through exploring the mechanism of Mff expression regulation, we found that HIF-1 $\alpha$ binds directly to and regulates Mff in the HNSCC cell cytoplasm. Through further experiments, the results revealed that hypoxia-induced ROS increased HIF-1 $\alpha$ /Mff levels, promoting mitochondrial fission and CDDP sensitivity (Figure 7D). Emerging evidence suggests that abnormal mitochondrial morphology is relevant in various diseases. ${ }^{25,26}$ Moreover, the biological mechanisms of the response of mitochondrial fission to CDDP sensitivity in malignant tumors are still unclear. Previous studies have revealed that an increase in mitochondrial fission in cancer cells enhances apoptosis and CDDP sensitivity in gastric cancers, ${ }^{27}$ gynecological cancers, ${ }^{28}$ and tongue squamous carcinomas. ${ }^{18,19}$ Due to the hypoxic microenvironment of HNSCCs, the cells were cultured under hypoxic conditions in the present study. A recent study showed that hypoxia-induced mitochondrial fission causes CDDP resistance in ovarian cancer cells. ${ }^{8}$ In the present study, our findings indicated that hypoxia-induced mitochondrial fission promotes CDDP sensitivity in HNSCC cells. The opposite effects of tumor cell mitochondrial fission on CDDP sensitivity may be explained by differences in the cancer cells.

Carefully orchestrated interactions between mitochondrial proteins that facilitate tumor cell death remain an immediate focus of research. Mff, a key molecule that determines mitochondrial size and shape and the regulation of mitochondrial cell death, is a novel therapeutic target in cancer. ${ }^{29,30}$ LATS2 facilitates the apoptosis of non-small cell lung cancer by regulating Mff-induced mitochondrial fission. ${ }^{31}$ Furthermore, Mff-induced mitochondrial fission was attributed to decreased colorectal cancer cell viability. ${ }^{32}$ Our findings showed that Mff was a key molecule participating in mitochondrial fission, contributing to CDDP sensitivity in hypoxia-cultured HNSCC cells. Both Mff and Mfn2 expression were upregulated in HNSCC tissues, indicating that mitochondrial dynamics in tumor tissues are more active than that in normal tissues. Moreover, the expression of Mff and HIF-1 $\alpha$ in HNSCC patients showed that hypoxia-induced mitochondrial fission events in HNSCC are correlated with chemosensitivity. Recent evidence has shown that HIF- $1 \alpha$ regulates the participation of mitochondrial dynamics in various biological behaviors. ${ }^{33,} 34,35$ Under hypoxic conditions, HIF-1 $\alpha$ modulated mitochondrial fission by direct regulation of the expression of Drp $1 .{ }^{36}$ Due to the markedly positive correlation between HIF-1 $\alpha$ and Mff, further experiments in the present study revealed that HIF- $1 \alpha$ participates in mitochondrial fission by regulating Mff.

Previous studies have revealed that hypoxic conditions could promote an increased level of ROS in the tumor microenvironment. ${ }^{8}, 11$ The ROS are generated by mitochondria and other cellular elements and participate in malignant tumor biological behavior through the regulation of HIF-1 $\alpha^{10,37,38}$ Hypoxia-induced mitophagy is associated with cancer aggressiveness via the interplay of ROS/HIF-1 $\alpha$ in gastric cancer cells. ${ }^{39}$ Our results demonstrated that hypoxia-induced ROS increases the expression of HIF- $1 \alpha$ and Mff, while NAC attenuates these ROS effects.

In summary, we demonstrated that hypoxia-induced ROS promote mitochondrial fission and CDDP chemosensitivity via HIF-1 $\alpha / \mathrm{Mff}$ regulation in HNSCC cells. Moreover, Mff may serve as a new biomarker for the prediction of neoadjuvant chemosensitivity and is important for overcoming chemoresistance, which requires further exploration. 


\section{Acknowledge}

This work was supported by the Youth Program of the Natural Science Foundation of China (81902808).

\section{Conflict of interest}

There is no any potential conflict of interest. The authors do not have commercial or other associations that might pose a conflict of interest.

\section{Reference}

1. Kamangar F, Dores GM, Anderson WF. Patterns of cancer incidence, mortality, and prevalence across five continents: defining priorities to reduce cancer disparities in different geographic regions of the world. J Clin Oncol 2006, 24(14): 2137-2150.

2. Wu K, Lei JS, Mao YY, Cao W, Wu HJ, Ren ZH. Prediction of Flap Compromise by Preoperative Coagulation Parameters in Head and Neck Cancer Patients. J Oral Maxillofac Surg 2018, 76(11): 2453 e2451-2453 e2457.

3. Leemans CR, Braakhuis BJ, Brakenhoff RH. The molecular biology of head and neck cancer. Nat Rev Cancer 2011, 11(1): 9-22.

4. Jacinto JK, Co J, Mejia MB, Regala EE. The evidence on effectiveness of weekly vs triweekly cisplatin concurrent with radiotherapy in locally advanced head and neck squamous cell carcinoma (HNSCC): a systematic review and meta-analysis. The British journal of radiology 2017, 90(1079): 20170442.

5. Brumwell A, Fell L, Obress L, Uniacke J. Hypoxia influences polysome distribution of human ribosomal protein $\mathrm{S} 12$ and alternative splicing of ribosomal protein mRNAs. RNA (New York, NY) 2020.

6. Gao L, Dou ZC, Ren WH, Li SM, Liang X, Zhi KQ. CircCDR1as upregulates autophagy under hypoxia to promote tumor cell survival via AKT/ERK(1/2)/mTOR signaling pathways in oral squamous cell carcinomas. Cell Death Dis 2019, 10(10): 745.

7. Carreau A, El Hafny-Rahbi B, Matejuk A, Grillon C, Kieda C. Why is the partial oxygen pressure of human tissues a crucial parameter? Small molecules and hypoxia. Journal of cellular and molecular medicine 2011, 15(6): 1239-1253.

8. Han Y, Kim B, Cho U, Park IS, Kim SI, Dhanasekaran DN, et al. Mitochondrial fission causes cisplatin resistance under hypoxic conditions via ROS in ovarian cancer cells. Oncogene 2019, 38(45): 7089-7105.

9. Wiechec E, Hansson KT, Alexandersson L, Jonsson JI, Roberg K. Hypoxia Mediates Differential Response to Anti-EGFR Therapy in HNSCC Cells. Int J Mol Sci 2017, 18(5).

10. Prasad S, Gupta SC, Tyagi AK. Reactive oxygen species (ROS) and cancer: Role of antioxidative nutraceuticals. Cancer Lett 2017, 387: 95-105.

11. Tretter L, Patocs A, Chinopoulos C. Succinate, an intermediate in metabolism, signal transduction, ROS, hypoxia, and tumorigenesis. Biochimica et biophysica acta 2016, 1857(8): 1086-1101.

12. Kim J, Barsoum IB, Loh H, Pare JF, Siemens DR, Graham CH. Inhibition of Hypoxia-Inducible Factor 1 Alpha Accumulation by Glyceryl Trinitrate and Cyclic Guanosine Monophosphate. Bioscience reports 2020. 
13. Zhang J, Zhang Q, Lou Y, Fu Q, Chen Q, Wei T, et al. Hypoxia-inducible factor1alpha/interleukin-1beta signaling enhances hepatoma epithelial-mesenchymal transition through macrophages in a hypoxic-inflammatory microenvironment. Hepatology 2018, 67(5): 1872-1889.

14. Cui CP, Wong CC, Kai AK, Ho DW, Lau EY, Tsui YM, et al. SENP1 promotes hypoxia-induced cancer stemness by HIF-1alpha deSUMOylation and SENP1/HIF-1alpha positive feedback loop. Gut 2017, 66(12): 2149-2159.

15. Suen DF, Norris KL, Youle RJ. Mitochondrial dynamics and apoptosis. Genes Dev 2008, 22(12): 1577-1590.

16. Chen H, Chan DCJCM. Mitochondrial Dynamics in Regulating the Unique Phenotypes of Cancer and Stem Cells. 26(1): 39-48.

17. Han Y, Cho U, Kim S, Park IS, Cho JH, Dhanasekaran DN, et al. Tumor microenvironment on mitochondrial dynamics and chemoresistance in cancer. 2018: 1-411.

18. Tian T, Lv X, Pan G, Lu Y, Chen W, He W, et al. Long Noncoding RNA MPRL Promotes Mitochondrial Fission and Cisplatin Chemosensitivity via Disruption of Pre-miRNA Processing. Clin Cancer Res 2019, 25(12): 3673-3688.

19. Fan S, Tian T, Chen W, Lv X, Lei X, Zhang H, et al. Mitochondrial miRNA Determines Chemoresistance by Reprogramming Metabolism and Regulating Mitochondrial Transcription. Cancer Res 2019, 79(6): 1069-1084.

20. Chen Q, Wu K, Qin X, Yu Y, Wang X, Wei K. LASP1 promotes proliferation, metastasis, invasion in head and neck squamous cell carcinoma and through direct interaction with HSPA1A. Journal of cellular and molecular medicine 2019.

21. Kashatus DF, Lim KH, Brady DC, Pershing NL, Cox AD, Counter CM. RALA and RALBP1 regulate mitochondrial fission at mitosis. Nature cell biology 2011, 13(9): 1108-1115.

22. Kujan O, Shearston K, Farah CS. The role of hypoxia in oral cancer and potentially malignant disorders: a review. J Oral Pathol Med 2017, 46(4): 246-252.

23. Niu Y, Bao L, Chen Y, Wang C, Luo M, Zhang B, et al. HIF-2-induced long non-coding RNA RAB11B-AS1 promotes hypoxia-mediated angiogenesis and breast cancer metastasis. Cancer Res 2020 .

24. Lee H, Yoon Y. Mitochondrial fission and fusion. Biochemical Society transactions 2016, 44(6): 1725-1735.

25. Molnar MJ, Kovacs GG. Mitochondrial diseases. Handbook of clinical neurology 2017, 145: 147-155.

26. Bose A, Beal MF. Mitochondrial dysfunction in Parkinson's disease. Journal of neurochemistry 2016, 139 Suppl 1: 216-231.

27. Li B, Wang W, Li Z, Chen Z, Zhi X, Xu J, et al. MicroRNA-148a-3p enhances cisplatin cytotoxicity in gastric cancer through mitochondrial fission induction and cyto-protective autophagy suppression. S0304383517305967.

28. Kong B, Tsuyoshi H, Orisaka M, Shieh DB, Yoshida Y, Tsang BK. Mitochondrial dynamics regulating chemoresistance in gynecological cancers. Annals of the New York Academy of Sciences 2015, 1350: 1-16.

29. Seo JH, Chae YC, Kossenkov AV, Lee YG, Tang HY, Agarwal E, et al. MFF Regulation of 
Mitochondrial Cell Death Is a Therapeutic Target in Cancer. Cancer Res 2019, 79(24): 62156226.

30. Seo JH, Agarwal E, Chae YC, Lee YG, Garlick DS, Storaci AM, et al. Mitochondrial fission factor is a novel Myc-dependent regulator of mitochondrial permeability in cancer. EBioMedicine 2019, 48: 353-363.

31. Xie Y, Lv Y, Zhang Y, Liang Z, Han L, Xie Y. LATS2 promotes apoptosis in non-small cell lung cancer A549 cells via triggering Mff-dependent mitochondrial fission and activating the JNK signaling pathway. Biomedicine \& pharmacotherapy = Biomedecine \& pharmacotherapie 2019, 109: 679-689.

32. Jieensinue S, Zhu H, Li G, Dong K, Liang M, Li Y. Tanshinone IIA reduces SW837 colorectal cancer cell viability via the promotion of mitochondrial fission by activating JNK-Mff signaling pathways. BMC cell biology 2018, 19(1): 21 .

33. He Y, Fang X, Shi J, Li X, Xie M, Liu X. Apigenin attenuates pulmonary hypertension by inducing mitochondria-dependent apoptosis of PASMCs via inhibiting the hypoxia inducible factor 1alpha-KV1.5 channel pathway. Chemico-biological interactions 2020: 108942.

34. Li HS, Zhou YN, Li L, Li SF, Long D, Chen XL, et al. HIF-1alpha protects against oxidative stress by directly targeting mitochondria. Redox biology 2019, 25: 101109.

35. Mylonis I, Kourti M, Samiotaki M, Panayotou G, Simos G. Mortalin-mediated and ERKcontrolled targeting of HIF-1alpha to mitochondria confers resistance to apoptosis under hypoxia. Journal of cell science 2017, 130(2): 466-479.

36. Chen X, Yao JM, Fang X, Zhang C, Yang YS, Hu CP, et al. Hypoxia promotes pulmonary vascular remodeling via HIF-1alpha to regulate mitochondrial dynamics. Journal of geriatric cardiology : JGC 2019, 16(12): 855-871.

37. Fuhrmann DC, Brune B. Mitochondrial composition and function under the control of hypoxia. Redox biology 2017, 12: 208-215.

38. Cheng J, Yang HL, Gu CJ, Liu YK, Shao J, Zhu R, et al. Melatonin restricts the viability and angiogenesis of vascular endothelial cells by suppressing HIF-1alpha/ROS/VEGF. International journal of molecular medicine 2019, 43(2): 945-955.

39. Shida M, Kitajima Y, Nakamura J, Yanagihara K, Baba K, Wakiyama K, et al. Impaired mitophagy activates mtROS/HIF-1alpha interplay and increases cancer aggressiveness in gastric cancer cells under hypoxia. Int J Oncol 2016, 48(4): 1379-1390. 